\title{
A numerical study of the role of vertical structure of vorticity during tropical cyclone genesis
}

\author{
T. N. Venkatesh ${ }^{\mathrm{a}, 1}$, Joseph Mathew ${ }^{\mathrm{b}}$ \\ ${ }^{a}$ Flosolver Unit, National Aerospace Laboratories, PB 1779, Bangalore 560017, India \\ ${ }^{b}$ Department of Aerospace Engineering, Indian Institute of Science, Bangalore 560012, \\ India
}

\begin{abstract}
An eight-level axisymmetric model with simple parameterizations for clouds and the atmospheric boundary layer was developed to examine the evolution of vortices that are precursors to tropical cyclones. The effect of vertical distributions of the vorticity, especially that arising from a merger of mid-level vortices, has been studied to provide support for a new vortex-merger theory of tropical cyclone genesis. The basic model was validated with the analytical results available for the spin-down of axisymmetric vortices. With the inclusion of the cloud and boundary layer parameterizations, the evolution of deep vortices into hurricanes and the subsequent decay is simulated quite well. Effects of several parameters such as initial vortex strength, radius of maximum winds, sea surface temperature and latitude (Coriolis parameter) on the evolution were examined.

A new finding is the manner in which mid-level vortices of the same strength decay and how, on simulated merger of these mid-level vortices, the resulting vortex amplifies to hurricane strength in a realistic time-frame. The importance of the sea-surface temperature on evolution of full vortices has been studied and explained. Also it is found that the strength of the surface vortex determines the time taken by the deep vortex to amplify to hurricane strength.
\end{abstract}

Key words:

Tropical cyclone, genesis, balanced vortex model, atmospheric vorticity

Email addresses: tnv@flosolver.nal.res.in (T. N. Venkatesh), joseph@aero.iisc.ernet.in (Joseph Mathew)

${ }^{1}$ Corresponding author, Tel: +91-80-25086594 
evolution

\section{Introduction}

Tropical cyclones (TC), also called hurricanes, are large three-dimensional vortices in the atmosphere. Computational models are very useful tools for studying these systems since direct controlled experiments are not possible and laboratory simulations are difficult. A wide variety of models of different resolution and sophistication are used for basic TC studies. These range from high resolution general circulation models (GCM), regional models such as MM5, RAMS, WRF, and simple three level models. With the availability of increasing computing power, the tendency to use bigger models such as MM5/WRF is natural. For example, Fudeyasu et al (2008) have successfully simulated the lifecycle of tropical cyclones using the global, nonhydrostatic, cloud-system-resolving model NICAM at a horizontal resolution of $7 \mathrm{~km}$. At the same time use of simpler three-layer models continues because they can throw light on the important processes (Emanuel, 1989, Zhu et al 2001, Zhu and Smith, 2002). The attempt is to retain the minimum number of relevant features to get a reasonable picture.

The authors have proposed a model of TC genesis (Venkatesh 2003, Venkatesh and Mathew, 2004) in which mesoscale mid-level vortices (termed $\mathrm{MCV}$, for mesoscale convectively-generated vortices) play a crucial role in the early stages. In this model, the early stages consist of mid-level MCVs which interact. This process is largely two-dimensional and non-axisymmetric. The later stages consist of the larger merged vortex, which extends down to the boundary layer. We consider the initial evolution to be "top-down" in a manner similar to that proposed by others (Ritchie and Holland 1997, Bister and Emanuel 1997). Subsequent development of the merged vortex takes place in an essentially axisymmetric manner. The other view of TC genesis is the "bottom-up" approach suggested by Hendricks et al. 2004 and Montgomery et al. 2006. Interaction of mesoscale vortices prior to TC genesis have been observed in the Pacific (Ritchie and Holland, 1997) and the Bay of Bengal (Venkatesh, 2006). Some of the important issues which can be elucidated from numerical simulations are i) the difference in the evolution of mid-level MCVs and deep vortices which extend down to the boundary layer, ii) evolution of MCVs following merger and iii) the effect a weak surface vortex has on mid-level vortices. The objective of this study is to answer these questions. 
To simulate the essential features of this process, a multi-level model is required. Therefore an eight-level, axisymmetric, balanced vortex model was developed. The main features of this model are that it solves the hydrostatic, balanced flow equations with simple parameterizations of the boundary layer and clouds. Also, the height is used as a co-ordinate instead of the pressure (or sigma) as is common in many atmospheric models. An advantage of using height is that the location and extent of the mid-level vortices can be specified exactly. The need to develop another simple hurricane model arose because, most of the simple models which have been developed (Zhu et al 2001, 2002), including those available in the public domain (Emanuel, 1995), have been three-layer models. In a three-layer model the cloud parametrization is done in a very simple manner, keeping the essential physics required for studying certain effects. Such a model would not be appropriate for our study since we are interested in mid-level vortices and their extension to the boundary layer, as well as finite-amplitude effects. Therefore a model with 8 layers, which are sufficient to resolve the vertical structure, was developed. The model is similar to Sundqvist's (1970) ten-level hurricane model which has been used extensively by Challa et al $(1980,1998)$ for many studies. The model of the boundary layer cumulus mass flux is similar to Emanuel's model based on quasi-equilibrium.

The structure of the paper is as follows: Formulation of the model equations is described section 2. The numerics, computational and details of the validation with semi-analytical results are provided in section 3. Section 4 contains the main results of the simulations carried out. Studies with deep vortices, mid-level vortices and the parameter sweep experiments are described. Conclusions are presented in section 5 .

\section{Model formulation}

As stated in the introduction, three-layer models are not adequate for our study since we are interested in mid-level vortices and their extension to the boundary layer. To represent the vertical structure, a sufficient number of layers, at least 5, are required. The present model formulation can handle an arbitrary number of levels. However, to keep the model economical, eight levels are used.

To obtain a reasonable representation of the flow structure and physics, an axisymmetric, hydrostatic balanced vortex model is used with the effects of clouds, radiation, boundary layer and sea-surface parametrized in a sim- 
ple manner. The balanced vortex approximation was first used by Eliassen (1952) for study of meridional circulations. It has since been used extensively in hurricane related studies (Schubert and Hack, 1982, 1983) and also for studies of idealized monsoon systems (Wirth, 1998). The main assumptions are that the radial flow is in a state of gradient wind balance and that there is hydrostatic balance in the vertical. Together, they imply that the azimuthal velocity and temperature perturbations are related by the thermal wind equation. Also, the secondary flow in the vertical plane can be obtained by solving a generalized Poisson equation.

The domain was divided into the surface boundary layer and the outer region. The boundary layer was considered to be the sub-cloud layer and to have a constant depth $h_{B L}$. The outer region extended from the top of the boundary layer to a height $z_{\max }$.

The prognostic equations in the interior are for the azimuthal velocity $v$ and the saturation equivalent potential temperature $\theta_{e}^{*}$ (which is nearly a conserved quantity).

$$
\begin{gathered}
\frac{\partial v}{\partial t}=-u \eta-w \frac{\partial v}{\partial z}+D_{v} \\
\frac{\partial \theta_{e}^{*}}{\partial t}=-u \frac{\partial \theta_{e}^{*}}{\partial r}-w \frac{\partial \theta_{e}^{*}}{\partial z}+D_{\theta}+\dot{H}_{r a d}
\end{gathered}
$$

Here $u$ is the radial velocity, $w$ the vertical velocity, $D_{v}$ represents the diffusion of $v$ (modelled), $\eta=f+\zeta=f+v / r+\partial v / \partial r$ is the absolute vorticity, $f$ is the Coriolis parameter due to earth's rotation, $D_{\theta}$ represents the diffusion of $\theta_{e}^{*}$ and $\dot{H}_{r a d}$ the radiative cooling term. In Sundqvist's model, specific humidity is used as the second prognostic variable. Using $\theta_{e}^{*}$ instead has the advantage that explicit treatment of condensation is not required in the prognostic equations (heating due to the condensation term appears in the cloud model as will be described later). A mean value of the virtual potential temperature, $\overline{\theta_{v}}$ was defined which is a function of $z$ only. Perturbations of the virtual potential temperature $\left(\theta_{v}=\theta[1+0.61 q]\right)$, from the mean value $\overline{\theta_{v}}$ were related to $v$ by the thermal wind equation

$$
\frac{\overline{\theta_{v}}}{\bar{\rho} g} \frac{\partial}{\partial z}\left[\frac{\bar{\rho} v^{2}}{r}+\bar{\rho} f v\right]=\frac{\partial \theta_{v}}{\partial r} .
$$

Given $v$, this equation can be solved for $\theta_{v}$. An additional constraint is that the values of $\theta_{v}$ should be less than $\theta_{v}^{*}$ (the saturation value) which was calculated from $\theta_{e}^{*}$ and the pressure $p$. 
The perturbation pressure was obtained from the gradient wind relation

$$
\frac{1}{\rho} \frac{\partial p}{\partial r}=\frac{v^{2}}{r}+f v
$$

The flow in the $r-z$ plane was obtained by solving a Poisson equation for the streamfunction $\psi$

$$
\frac{\partial}{\partial r}\left[A \frac{\partial \psi}{\partial r}+B \frac{\partial \psi}{\partial z}\right]+\frac{\partial}{\partial z}\left[C \frac{\partial \psi}{\partial r}+D \frac{\partial \psi}{\partial z}\right]=\frac{\partial S_{q}}{\partial r}+\frac{\partial S_{f}}{\partial z}
$$

where

$$
\begin{gathered}
A=-\frac{1}{r} \frac{g}{\bar{\theta}} \frac{\partial \overline{\theta_{v}}}{\partial z}, \quad B=\frac{1}{\bar{\rho} r} \frac{\partial}{\partial z}\left[\frac{\bar{\rho} v^{2}}{r}+\bar{\rho} f v\right], \quad C=\left(\frac{2 v}{r}+f\right) \frac{1}{r} \frac{\partial v}{\partial z} \\
D=-\left(\frac{2 v}{r}+f\right) \frac{1}{r}\left(\frac{\partial v}{\partial r}+\frac{v}{r}+f\right) \\
S_{q}=\left(\frac{\bar{\rho} g \dot{Q}}{C_{p} \bar{T}}\right), \quad S_{f}=-\left(\frac{2 v}{r}+f\right) D_{v} .
\end{gathered}
$$

Boundary conditions for solving the $\psi$ are needed at lower, upper, inner and outer boundaries. They were prescribed as follows: i) at $r=0$ and $z=z_{\max }, \psi=0$, ii) Lower domain, i.e., at $z=h_{B L} \psi$ is calculated from the boundary layer model, and iii) at $r=r_{\max }$, extrapolation boundary conditions were used.

The velocity components $u$ and $w$ were obtained from the streamfunction:

$$
u=\frac{1}{\bar{\rho} r} \frac{\partial \psi}{\partial z} \quad w=-\frac{1}{\bar{\rho} r} \frac{\partial \psi}{\partial r}
$$

A detailed derivation of the above equation (5) can be found in Appendix C of Venkatesh (2003).

Models are needed for the diffusion terms $D_{v}$ and $D_{\theta}$ and radiation $\dot{H}_{\text {rad }}$ in eqn. (1) and (2), heating rate $\dot{Q}$ in the source term $S_{q}$ of eqn. (5), and $\psi$ and $\theta_{v}$ at the top of the boundary layer. The diffusion terms were obtained from the simple eddy viscosity model:

$$
D_{v}=\frac{\partial}{\partial z}\left(\tau_{\phi z}\right)+\frac{1}{r^{2}} \frac{\partial}{\partial r}\left(r^{2} \tau_{\phi r}\right)
$$


with

$$
\tau_{\phi z}=K_{V} \frac{\partial v}{\partial z}, \quad \tau_{\phi r}=K_{H}\left(\frac{\partial v}{\partial r}-\frac{v}{r}\right)
$$

and

$$
D_{\theta}=\frac{\partial}{\partial z}\left(K_{V} \frac{\partial \theta_{e}^{*}}{\partial z}\right)+\frac{1}{r^{2}} \frac{\partial}{\partial r}\left(r^{2} K_{H} \frac{\partial \theta_{e}^{*}}{\partial r}\right)
$$

where $K_{H}$ and $K_{V}$, the eddy diffusivities (assumed to be the same for both momentum and heat transfer), were calculated from the boundary layer model, and are functions of $r$ and $z$. The radiation term

$$
\dot{H}_{r a d}=-\frac{\theta_{e}^{\prime}}{\tau_{r a d}}
$$

where $\tau_{\text {rad }}$ is a decay time of the order of 24 hours.

\subsection{Boundary Layer model}

The boundary layer is modelled by a single layer in which the evolution equation solved for $\theta_{e}$ is

$$
\frac{\partial \theta_{e}}{\partial t}=-u \frac{\partial \theta_{e}}{\partial r}-w_{m} \frac{\partial \theta_{e}}{\partial z}+D_{\theta}+\dot{H}_{r a d} .
$$

The azimuthal velocity is assumed to decrease linearly from its value at the top of the boundary layer, to the top of the surface layer by $20 \%$ (Montgomery et al 2001). The radial inflow velocity $u_{B L}$ is deduced in a manner similar to Ooyama (1969) and Emanuel (1986). In eqn. (1) for $v$ in the boundary layer, the major balance is between the diffusion term and the radial advection term. So,

$$
u_{B L} \eta=-\frac{C_{D}|v| v}{h_{B L}} .
$$

In regions of strong convective activity, vertical velocity can become large and the vertical convection term is included. Otherwise the computed values of $u$ can become unrealistic. Therefore in cloud regions, the following expression is used.

$$
u_{B L} \eta=-\frac{C_{D}|v| v}{h_{B L}}-w_{B L} \frac{\partial v}{\partial z}
$$

In the lower part of the boundary layer, the surface layer, Deacon's formula is used to calculate the aerodynamic drag coefficient

$$
C_{D}=1.1 \times 10^{-3}+4.0 \times 10^{-5} \mathrm{~V} .
$$


Here $\mathrm{V}$ is the speed at the top of the surface layer $(10 \mathrm{~m})$ in metres per second. This formula is probably the most widely used one in models of this class (Ooyama 1969, Montgomery et al 2001).

Matching of the fluxes at the top of the boundary layer leads to estimates of the eddy diffusivities

$$
\begin{gathered}
K_{V_{B L}}=C_{D} * V / h_{B L}, \\
K_{H_{B L}}=K_{V_{B L}} *\left(l_{h} / l_{v}\right)^{2} .
\end{gathered}
$$

with typical values of $l_{h}$ and $l_{v}$ as 2000 and 200, respectively. In the interior, the eddy viscosities decay with height $z$ above the boundary layer value by the factor $\exp \left(-a\left(z / z_{\max }\right)^{2}\right)$. Values of 3 to 10 for the constant $a$ did not cause any significant change in the results. Also, minimum values of 1 for $K_{V}$ and 100 for $K_{H}$ were prescribed.

The stream function at the top of the boundary layer is required for the interior solution. It is the sum of two parts: the Ekman pumping component and the additional cumulus mass flux in regions of convection. The Ekman pumping component $w_{c a}$, also called the clear air component, results from the induced radial velocity $u_{B L}$. Therefore

$$
\psi_{E k m a n}=\rho_{B L} u_{B L} h_{B L}
$$

\subsection{The cloud model}

Clouds are very important constituents of the atmosphere and the most difficult to model. An accurate representation of clouds requires covering a range of scales from the microphysical to the mesoscale. There are various degrees of approximation from one-dimensional models, to those with full microphysics (Houze 1993). Since incorporation of such details would increase the complexity and be inappropriate in our model, we model only the primary effects of cumulus clouds.

The cloud model in this study consists of a boundary layer part and an interior part. Grid points where the Ekman pumping is upward $(w>0)$ are considered to have deep cumulus clouds. In the boundary layer the cumulus mass flux is calculated by a formulation which is similar to Emanuel (1995). The equation for moist entropy in the boundary layer $s_{b}=C_{p} \ln \theta_{e}$ is

$$
\frac{d_{h} s_{b}}{d t}+\left(w_{c a}-\delta w_{c m}\right)\left(s_{m}-s_{b}\right) / h_{B L}=\frac{C_{D}\left|\mathbf{V}_{\mathbf{b}}\right|\left(k_{s}^{*}-k_{b}\right)}{T_{b}}+\dot{Q}_{r a d} / T_{b}
$$


where $\delta w_{c m}$ is the net cumulus updraft velocity (this is the difference between the updraft and downdraft velocities), $V_{b}$ the surface layer velocity, $k_{b}$ the moist enthalpy of the boundary layer, $k_{s}^{*}$ the saturation moist enthalpy at the sea surface $\dot{Q}_{\text {rad }}$ the radiative cooling term, $T_{b}$ the boundary layer temperature, $d_{h} / d t$ the horizontal derivative and $s_{m}$ the entropy at the "middle" level where downdrafts originate. The quasi-equilibrium assumption implies that the time derivative and the radiative cooling term are neglected. Then, the equilibrium cumulus updraft velocity

$$
\delta w_{c m e q}=\frac{C_{D}\left|\mathbf{V}_{\mathbf{b}}\right|\left(k_{s}^{*}-k_{b}\right)}{T_{b}}+w_{c a}
$$

The actual cumulus mass flux relaxes to this equilibrium value on a time scale $\tau_{m c}$ which is of the order of a few hours.

$$
\frac{\partial \delta w_{c m}}{\partial t}=\frac{\delta w_{c m e q}-\delta w_{c m}}{\tau_{m c}}
$$

In the interior, the local heating rate $\dot{Q}$ is determined by calculating the difference between the "cloud temperature" at that point and the local temperature. In terms of the model variables,

$$
\dot{Q}(r, z)=\xi\left(\theta_{v}^{C}-\theta_{v}\right)
$$

$\theta_{v}^{C}$ is the potential temperature of a parcel lifted moist adiabatically (keeping $\theta_{e}^{*}$ constant) from the top of the sub cloud layer. Therefore, $\theta_{v}^{C}$ is equal to $\theta_{v}^{*}$ calculated from $\theta_{e}^{*}$ at the lowest interior level and the local value of pressure. The parameter $\xi$ is found for each column by equating the column integral of $\dot{Q}$ to the enthalpy flux into the column from the boundary layer which in turn is mainly the transfer from the sea surface. The enthalpy flux is modelled as

$$
e_{f l u x}=\delta w_{m c} T_{B L} \frac{\Delta s}{h_{B L}}
$$

where

$$
\Delta s=\Delta\left(C_{p} \ln \theta_{e}\right)=C_{p} \frac{\Delta \theta_{e}}{\theta_{e}}
$$

and $\Delta \theta_{e}=\theta_{e s}^{*}-\theta_{e_{B L}}$. 


\section{Numerics and Simulation details}

The domain of calculation is in the $r-z$ plane, bounded by $r_{\max }$ and $z_{\max }$ respectively. The locations of the various quantities on the grid used are as shown in Fig. 1. In the staggered grid, $\theta_{e}^{*}, \theta_{v}$ and the other thermodynamic quantities are co-located with $v$ at the cell centre, while $u$ and $w$ are at the cell faces and the streamfunction $\psi$ is at the corners. The staggered grid used here is similar to the Arakawa C-grid. The advantage of using such a grid is that oscillations of pressure are suppressed. Moreover, application of the boundary conditions can be done exactly.

To start the calculation, initial fields of $v$ and $\theta_{e}^{*}$ have to be specified. The initial velocity field for a deep vortex was calculated using the following expression

$$
v(r, z)=\frac{2 v_{\max }\left(r / r_{\text {vmax }}\right)}{1+\left(r / r_{\text {vax }}\right)^{2}}\left[1-\frac{z}{z_{\max }}\right]
$$

For a Rankine vortex, the velocity increases linearly upto $r_{v \max }$ and then varies as $1 / r$. This profile is a smoothened approximation since, the velocity increases linearly for small $r / r_{v \max }$, decreases as $1 / r$ for large $r / r_{v \max }$ and varies smoothly in between. The maximum value is $v_{\max }$ at $r_{v \max }$. The velocity field initialization for a mid-level vortex is described in section 4.4. The initial values of $p, \theta$ and $\theta_{e}^{*}$ are from Jordan's mean tropical sounding (Jordan 1958). Initial values of $u$ and $w$ are set to zero.

The sequence of computations in each time step is as follows:

1. The prognostics equations for $v$ (eqn. 1) and $\theta_{e}^{*}$ (eqn. 2) are integrated using a second order time-stepping scheme. Details of the scheme are described below.

2. Pressure is calculated from the balance condition (eqn. 4) and perturbation $\theta_{v}$ from equation 3 .

3. Various parametrized quantities (eddy diffusion terms, heating rates from the cloud and radiation models) are calculated in the interior. The source terms for the Poisson equation and the prognostic equations are thus obtained.

4. Integration of the boundary layer model is done to obtain the boundary conditions for the Poisson equation and the prognostic equations.

5. The Poisson equation for the streamfunction $\psi$ (eqn. 5) is solved using a standard iteration scheme.

6. The radial and vertical velocities are calculated from $\psi$. 


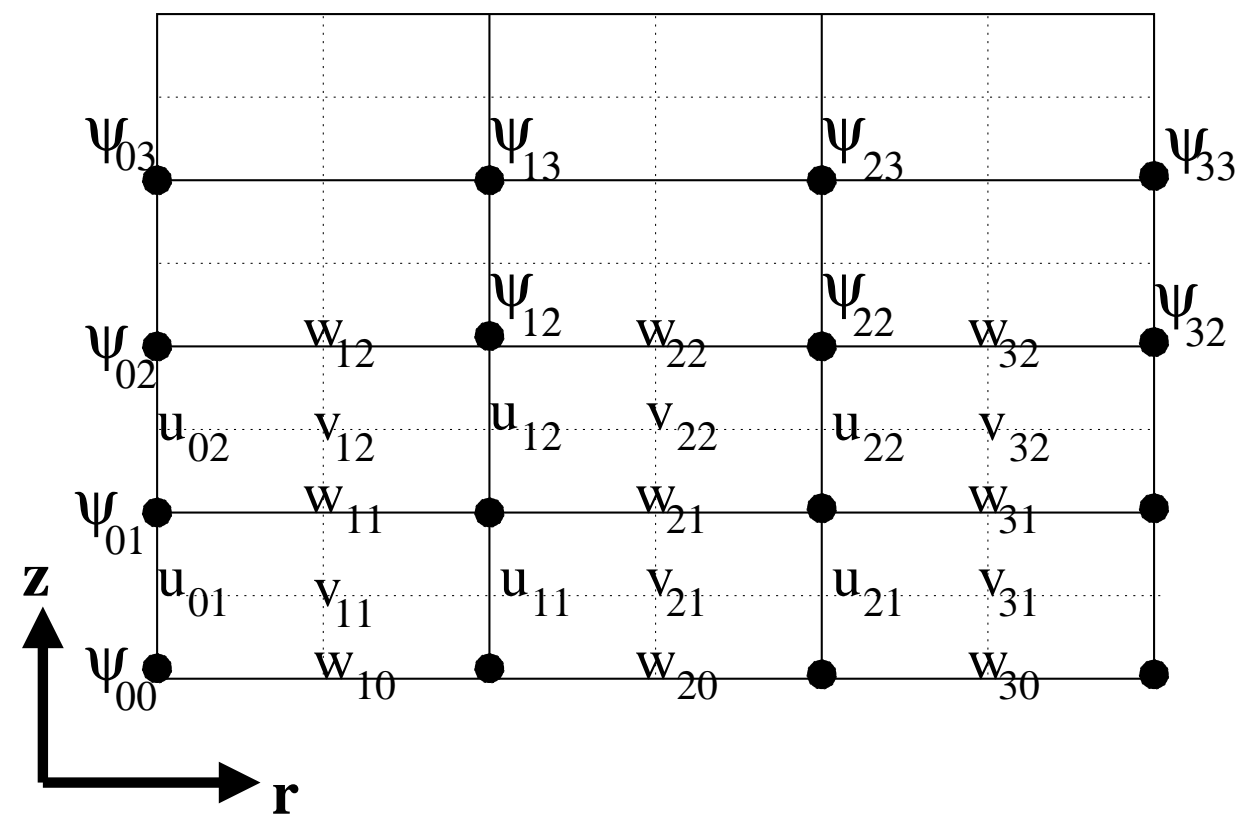

Figure 1: Schematic diagram of a portion of the staggered grid used for the computation. The azimuthal velocity $(v)$ is located at the cell centre, while the radial $(u)$ and vertical $(w)$ velocities are at cell faces. The stream-function $\psi$ is located at the corners of a cell. 
In this sequence of calculations, steps 1 and 5 are the most important. A mixed scheme (Kim and Moin 1985, Khalili et al 1997) of explicit timestepping for nonlinear terms (denoted NL) and implicit for diffusion terms was implemented for eqn.(1) as

$$
\frac{v^{n+1}-v^{n}}{\Delta t}=-\frac{1}{2}\left(3 N L^{n}-N L^{n-1}\right)+\frac{1}{2}\left(D_{v}^{n+1}+D_{v}^{n}\right)
$$

Approximate factorization of the diffusion terms leads to the form

$$
\left(I-\frac{\Delta t}{2} D_{z}\right)\left(I-\frac{\Delta t}{2} D_{r}\right) v^{n+1}=v^{n}-\frac{\Delta t}{2}\left(3 N L^{n}-N L^{n-1}\right)+\frac{\Delta t}{2}\left(D_{r}+D_{z}\right) v^{n},
$$

where $I$ is the identity operator, and terms of order $(\Delta t)^{2}$ have been dropped. Then, inversion of tridiagonal matrices in each direction suffices. The prognostic equation for $\theta_{e}^{*}(2)$ is treated similarly. A CFL condition for stability is

$$
\Delta t=\frac{h_{B L} \mathrm{CFL}}{V_{\max }} .
$$

Here, $\mathrm{CFL}=0.75$ was used.

The Poisson equation for $\psi$ was solved iteratively, with central differences used for evaluating the derivatives. For the other equations also, central differences are used for discretization.

The numerical parameters which can be varied in the model are given in table 1 . In addition there are flags for switching on the clouds, radiation and specifying a full or mid-level vortex in the initial field. The main differences between Sundqvist's model and the present model are as follows. i) the vertical co-ordinate $z$ is used here instead of pressure, ii) an evolution equation for $\theta_{e} *$ is used, not humidity $q$ and iii) the modelling of cloud heating is different.

\subsection{Validation of the numerics: decay of a deep vortex}

To test the dynamics and the numerics of the model/code, it was run with cloud terms switched off. Eliassen and Lystad (1977) studied a similar problem and arrived at an expression for the half life based on a simplified theory. Montgomery et al. (2001) have also used this expression to study the effect of drag parameterizations on the computed half lives. The expression for the half life is

$$
t_{1 / 2}=\frac{H-h}{\xi^{2} C_{D} V_{\text {initial }}}
$$




\begin{tabular}{|l|l|l|l|}
\hline Parameter & Symbol & Units & $\begin{array}{l}\text { Mean value } \\
\text { (Control) }\end{array}$ \\
\hline Domain size: horizontal & $R_{\max }$ & $\mathrm{km}$ & 500 \\
Domain size: vertical & $z_{\max }$ & $\mathrm{km}$ & 16.0 \\
Boundary Layer height & $h_{B L}$ & $\mathrm{~km}$ & 1.0 \\
Initial vortex strength & $v_{\max }$ & $\mathrm{m} / \mathrm{s}$ & 11.0 \\
Radius of maximum winds & $r_{\text {vmax }}$ & $\mathrm{km}$ & 90.0 \\
Sea surface temperature & $T_{\text {sea }}$ & $\mathrm{K}$ & 300.5 \\
Base latitude & & $\mathrm{deg}$ & 20 \\
Vortex height & & $\mathrm{km}$ & 16.00 \\
Radiation time constant & $\tau_{r a d}$ & $\mathrm{hrs}$ & 24.0 \\
Base latitude & & $\mathrm{degrees}$ & 20.0 \\
Cumulus mass flux time constant & $\tau_{m c}$ & $\mathrm{hrs}$ & 2.0 \\
Base drag coefficient in Deacon's formula & $C_{D 0}$ & - & $1.1 \times 10^{-3}$ \\
Velocity dependent part of drag & $C_{D r}$ & $\mathrm{~s} / \mathrm{m}$ & $4.0 \times 10^{-5}$ \\
\hline Time step & $\Delta_{t}$ & minutes & 5 \\
Integration time & $t_{\max }$ & hours & 240 \\
CFL number & $C F L$ & - & 0.75 \\
Number of cells in the $r$ direction & $n_{r}$ & - & 50 \\
Number of vertical layers & $n_{z}$ & - & 8 \\
\hline
\end{tabular}

Table 1: List of model and simulation parameters which can be varied in the code.

where $H$ is the height of the vortex, $h$ the boundary layer height, $C_{D}$ the drag coefficient, $V_{\text {initial }}$ the maximum initial azimuthal velocity and $\xi$ the reduction factor.

The computed and theoretical values of half lives are listed in Table 2. One can see that there is a reasonable agreement with the theory. This is a validation of the basic numerics of the code and also the range of eddy viscosities chosen. The differences could be due to the fact that in the theory an assumption is that the flow evolves to a state of cyclostrophic balance.

The decay of the maximum velocity is algebraic in time. For $V_{0}$ of 10 and 20 , velocities decay at the same rate throughout and the velocity maximum is at the surface at all times. For stronger and deeper vortices $\left(V_{0}=30,50\right.$, $H=10 \mathrm{~km}$ ), the vorticity near the surface decays at slightly faster rate and the velocity maximum is lifted up. This indicates that the eddy viscosities are not sufficient in the later stages. However since this happens after the velocity has reached a quarter of the initial value, we do not expect it to have 


\begin{tabular}{|c|r|r|r|}
\hline & \multicolumn{3}{|c|}{$t_{1 / 2}(\mathrm{hrs})$} \\
\hline$V_{\text {initial }}$ & Theory & \multicolumn{2}{|c|}{ Computed } \\
\cline { 3 - 4 }$(\mathrm{m} / \mathrm{s})$ & & $\mathrm{H}=5 \mathrm{~km}$ & $\mathrm{H}=10 \mathrm{~km}$ \\
\hline 10 & 115.0 & 101.70 & 111.32 \\
20 & 46.0 & 48.60 & 50.31 \\
30 & 25.0 & 28.00 & 28.95 \\
50 & 11.0 & 11.83 & 12.94 \\
\hline
\end{tabular}

Table 2: Comparison of computed half lives with the theory of Eliassen and Lystad for various initial vortex strengths, and with vortex heights $(H)$ of 5 and $10 \mathrm{~km}$

a significant impact on other simulations.

\begin{tabular}{|l|l|l|}
\hline Parameter & Units & Range \\
\hline Initial vortex strength: $V_{0}$ & $\mathrm{~m} / \mathrm{s}$ & $2,5,8,10,11$ and 12 \\
\hline Domain size: $R_{\max }$ & $\mathrm{km}$ & 500,1000 \\
\hline Radius of maximum winds & $\mathrm{km}$ & $50,60,70,80,90,100,125,150$ \\
\hline$T_{\text {sea }}$ & $\mathrm{K}$ & $298.5-302.5$ (in steps of 0.5$)$ \\
\hline Base latitude & $\mathrm{deg}$ & $15,16,17,18,19,20,22.5,25.0$ \\
\hline
\end{tabular}

Table 3: List of the experiments conducted with full vortices. The parameters changed and the values are listed.

The computational costs of running this model are quite low. For example, a ten day run with the full model (50 radial points and $\Delta t$ of 5 minutes) takes 4 minutes of real time on a desktop system with a $3 \mathrm{GHz}$ Intel Pentium IV processor and 1 GB of RAM.

\section{Studies with the full model}

The studies with the full model were of deep vortices whose velocity maxima in the vertical were at the top of the boundary layer, and mid-level vortices whose velocity maxima were at levels 3 or 4 .

A number of parameter studies were conducted using the model. A list is given in table 3 .

\subsection{Evolution of deep vortices}

The behavior of a deep vortex which extends from the top of the boundary layer to the top of the troposphere was studied by a series of simulations in 


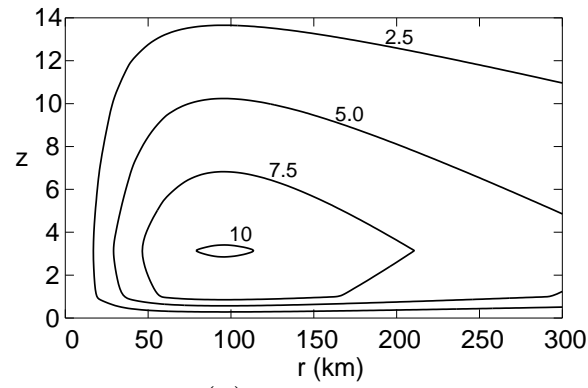

(a) $\mathrm{t}=0 \mathrm{hrs}$

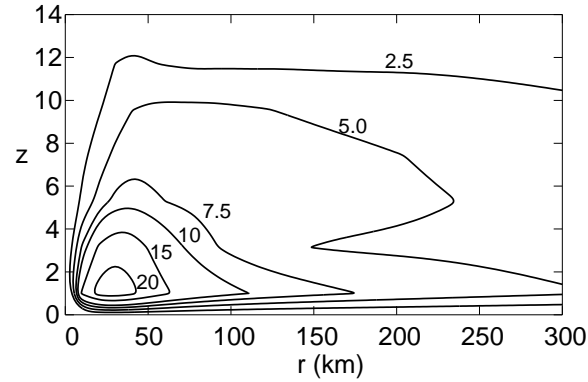

(c) $\mathrm{t}=24 \mathrm{hrs}$

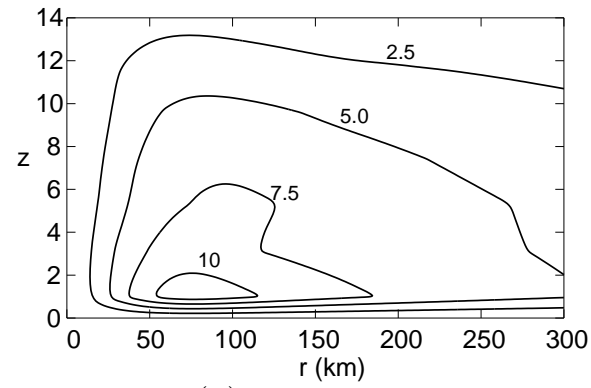

(b) $\mathrm{t}=12 \mathrm{hrs}$

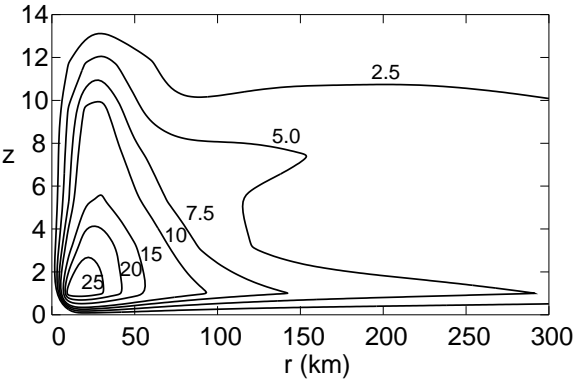

(d) $\mathrm{t}=48 \mathrm{hrs}$

Figure 2: Evolution of the vortex with an initial maximum velocity of $11 \mathrm{~m} / \mathrm{s}$. Azimuthal velocity contours are plotted at intervals of $2.5 \mathrm{~m} / \mathrm{s}$, starting from $2.5 \mathrm{~m} / \mathrm{s}$ upto $10 \mathrm{~m} / \mathrm{s}$ and at intervals of $5 \mathrm{~m} / \mathrm{s}$ from $10 \mathrm{~m} / \mathrm{s}$ onwards.

which the cloud model was turned on. The initial vortex had maximum velocity at the top of the boundary layer and decreased linearly with height, reaching zero at $z_{\max }$. The radial profile of azimuthal velocity was the same as that used for the decay studies (equation 12). The mean initial profiles for $\theta_{v}, \bar{p}$ and $\theta_{e}$ were taken from Jordan's sounding which has been used in many such studies (Ooyama 1969).

A typical run for which a hurricane lifecycle was simulated by the model consisted of the following parameters: $T_{\text {sea }}=300.5 \mathrm{~K}, r_{v \max }=90 \mathrm{~km}$, initial $V_{\max }=11 \mathrm{~m} / \mathrm{s}$. The variation of the velocity field with time is shown in Fig. 2. Initially the radius of maximum wind is at $90 \mathrm{~km}$. The initial wind is strong enough to cause large sea surface fluxes. This leads to increased heating and updraft mass flux in the clouds. This increases the radial flow in the boundary layer which increases the azimuthal velocity (term 1 in eqn. (1)). There are two main effects of the cloud. One is in increasing $u_{B L}$ and the other in lifting the streamlines (by vertical heating) and thus increasing the stretching. Initially, the streamlines consist of horizontal segments (inflow 


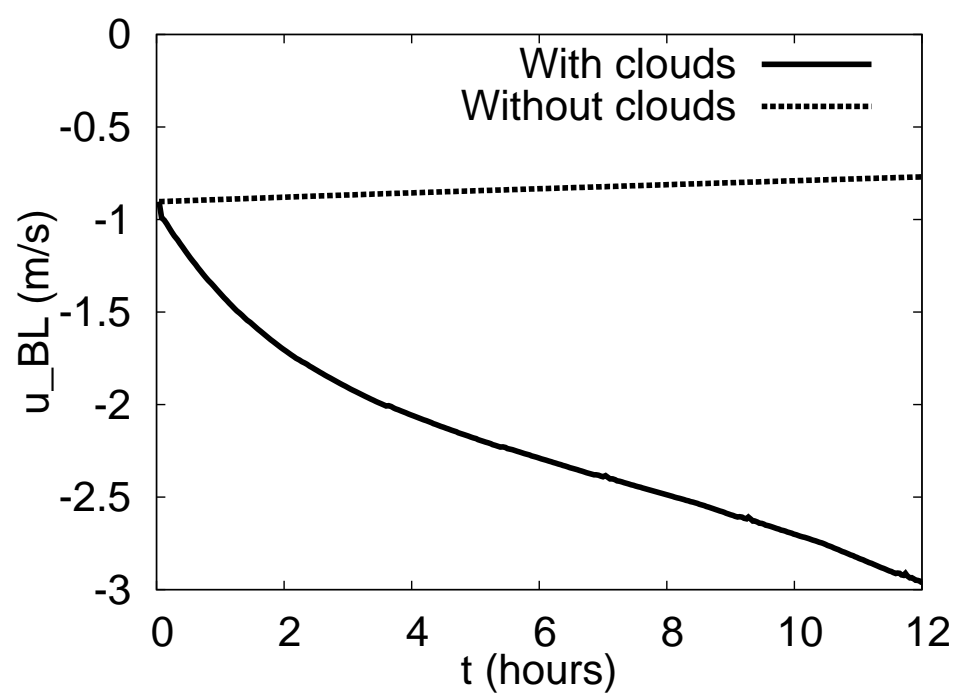

Figure 3: Radial flow in the boundary layer $\left(u_{B L}\right)$ with and without cloud heating as a function of time.

in the boundary layer and outflow at higher levels) connected by a curved section. As the vorticity intensifies, the vertical velocity gets established, with a larger values away from the surface (mid-levels), streamlines get lifted up due to advection by the vertical velocity.

The time evolution of the radial inflow with and without the cloud heating term is shown in figure 3. Without clouds, the radial inflow decreases and approaches zero as the vortex decays. The effect of clouds is to increase the inflow which in turn increases the vertical velocities.

Evolution of the heating rate due to clouds is shown in figure 4. One can see that the maxima moves inwards and upwards as time progresses. Also, the heating rates increase during the growth phase (48 hours). The interaction between azimuthal component of surface wind, sea-surface fluxes, cumulus heating at higher levels and secondary circulation constitutes a positive feedback cycle. Higher surface azimuthal winds $(v)$ increase the sea-surface fluxes, which in turn increase the cumulus heating at higher levels. This increases the secondary flow $(u, w)$ which leads to an increased azimuthal winds.

As the vortex amplifies, the radius of maximum winds decreases. Velocities also increase at the upper levels by both diffusion and advection. This positive feedback leads to rapid amplification of the vortex (Phase 1). The velocity reaches storm strength in 17 hours and hurricane strength in 25 


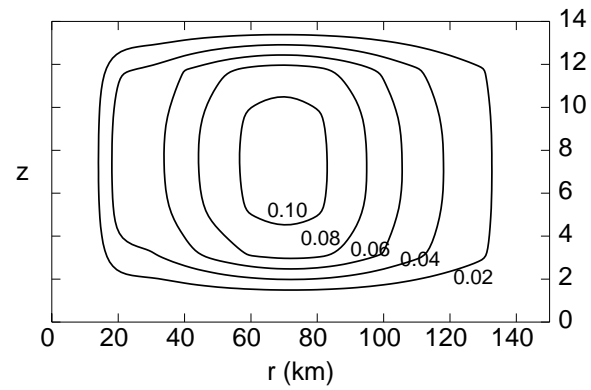

(a) $\mathrm{t}=12$

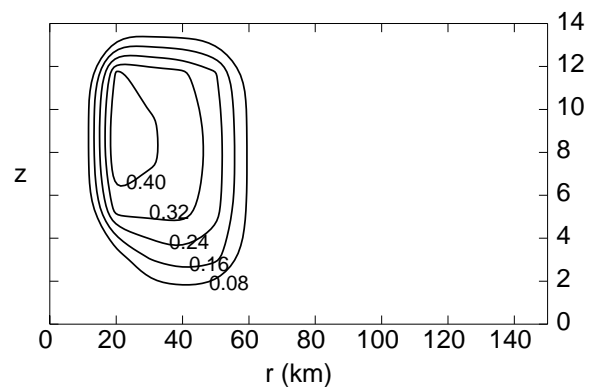

(c) $\mathrm{t}=24$

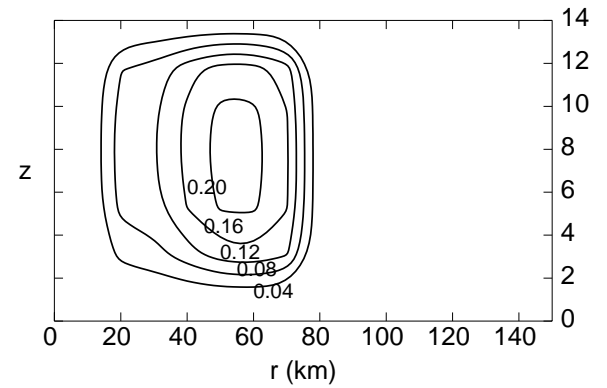

(b) $t=18$

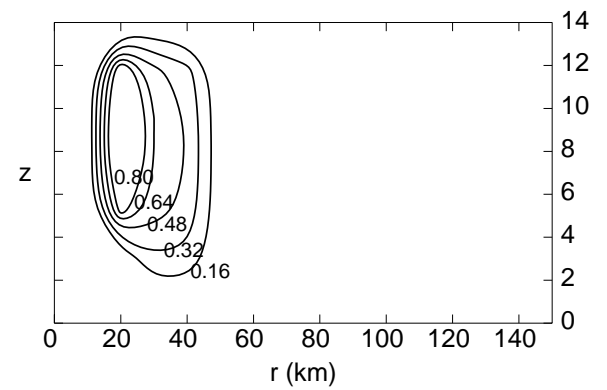

(d) $t=48$

Figure 4: Cross sections of the heating rate for integration started with initial $V_{\max }=11$ $\mathrm{m} / \mathrm{s}$ at (a) $\mathrm{t}=12 \mathrm{hrs},(\mathrm{b}) \mathrm{t}=18 \mathrm{hrs}$, (c) $\mathrm{t}=24 \mathrm{hrs}$ and (d) $\mathrm{t}=48 \mathrm{hrs}$,

hours. Then there is slowing down of the increase as the dissipative processes increase (Phase 2). The velocity maxima is reached at 48 hours. There is a balance of the tendencies to intensify and decay, so that a near steady state is reached. Then there is the third phase which consists of a slow decay of the vortex. The rate of decay here is lower than that for the case with no clouds as the cloud heating opposes the decay.

The structure of the vortex when the maximum velocity is reached (48 hours) is shown in Fig. 5. Contours of the azimuthal velocity $v$ are shown in Fig. 5 (a). The maxima close to the centre and the decrease both radially and in the vertical can be seen. From the plot of the streamfunction, (Fig. 5 (b)), the secondary circulation can be seen. The radial inflow in the boundary layer, large upward velocities in the central portion and radial outflow at higher levels can be seen. The high amounts of moisture in the boundary layer, regions of heating in the centre can be inferred from the contour plots of the saturated equivalent potential temperature $\theta_{e}^{*}$ (Fig. 5 (c)). The warm core structure of the vortex is clearly seen from Fig. 5 (d), where the perturbation potential temperature $\left(\theta_{v}^{\prime}\right)$ is plotted. 


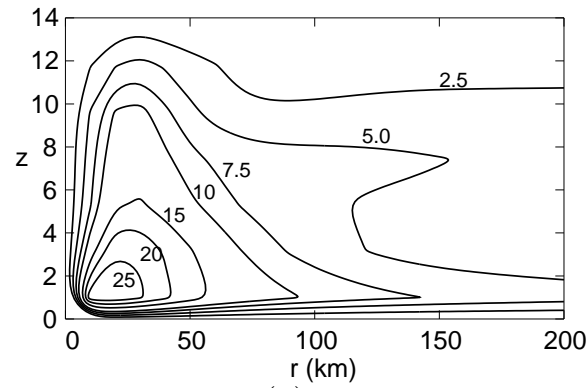

(a) $\mathrm{v}$

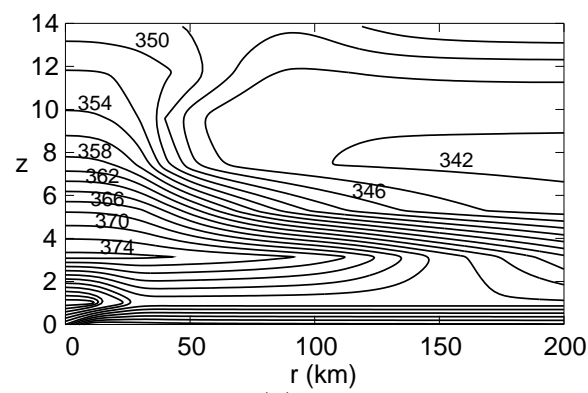

(c) $\theta_{e}^{*}$

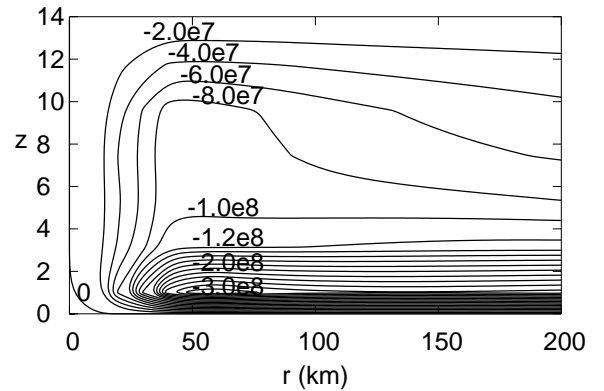

(b) $\psi$

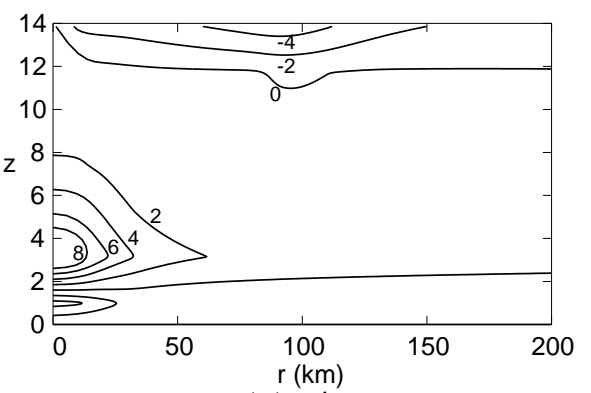

(d) $\theta_{v}^{\prime}$

Figure 5: Structure of the vortex at $\mathrm{t}=48$ hours for integration started with initial $V_{\text {max }}$ $=11 \mathrm{~m} / \mathrm{s}$. In the $r-z$ plane, contours are plotted for the following fields: (a) Azimuthal velocity $v(\mathrm{~m} / \mathrm{s})$, (b) Streamfunction, (c) Saturated equivalent potential temperature $\theta_{e}^{*}$ $(\mathrm{K})$ and $(\mathrm{d})$ Perturbation potential temperature $\theta_{v}^{\prime}(\mathrm{K})$

The radial profiles of azimuthal velocity are shown in Fig. 6. The growth is rapid with the maximum velocity increasing and the radius of maximum winds decreasing from $90 \mathrm{~km}$ initially to $20 \mathrm{~km}$ at 48 hours. The variation is qualitatively similar to that simulated by Sundqvist's model. The time taken to reach the maximum velocity is longer though (84 hours) in their case. In the decay phase, the shape of the radial profile is nearly the same. 


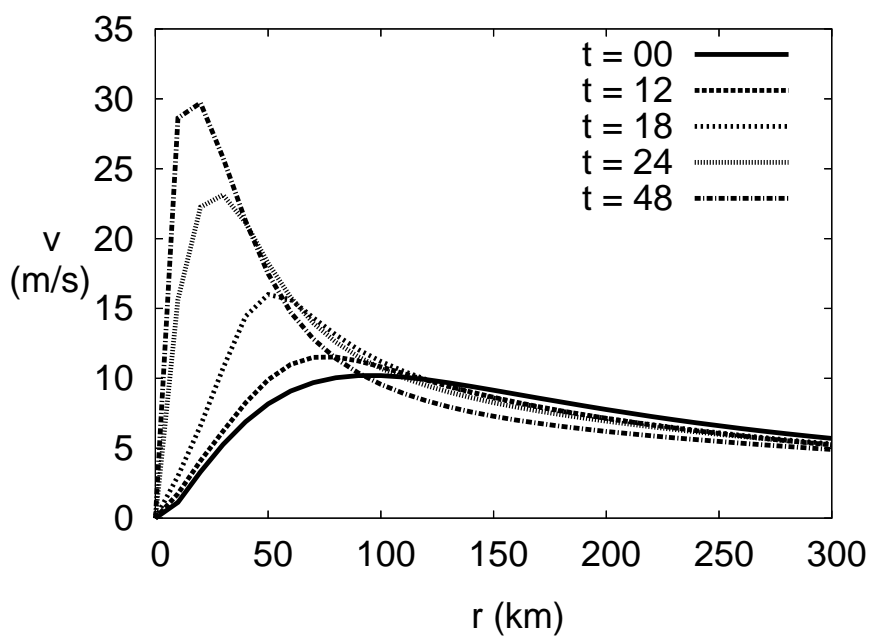

Figure 6: Evolution of the vortex with an initial maximum velocity of $11 \mathrm{~m} / \mathrm{s}$. Radial distribution of azimuthal velocity is plotted at different times (in hours) 


\subsection{Finite amplitude nature}

\begin{tabular}{|r|r|c|c|c|}
\hline $\begin{array}{r}V_{0} \\
(\mathrm{~m} / \mathrm{s})\end{array}$ & $\begin{array}{r}V_{\max } \\
(\mathrm{m} / \mathrm{s})\end{array}$ & $\begin{array}{r}t_{V \max } \\
(\mathrm{hrs})\end{array}$ & $\begin{array}{c}T_{\text {hurricane }} \\
(\mathrm{hrs})\end{array}$ & $\begin{array}{c}T_{\text {storm }} \\
(\mathrm{hrs})\end{array}$ \\
\hline 2.0 & 2.01 & 34.42 & - & - \\
5.0 & 11.98 & 86.63 & - & - \\
8.0 & 23.93 & 64.02 & - & 132 \\
10.0 & 32.93 & 49.87 & - & 220 \\
11.0 & 37.32 & 48.01 & 78 & 224 \\
12.0 & 41.90 & 48.01 & 118 & 227 \\
\hline
\end{tabular}

Table 4: Effect of initial vortex strength on the evolution of the system. $V_{\max }$ is the maximum velocity reached in the run, $t_{V_{\max }}$ is the time at which this velocity is reached, $T_{\text {hurricane }}$ the duration for which $v$ is above $33 \mathrm{~m} / \mathrm{s}$ and $T_{\text {storm }}$ the duration for which $v$ is above $17 \mathrm{~m} / \mathrm{s}$.

The finite-amplitude nature is evident on comparing simulations performed with different maximum initial velocities. The results are shown in Table 4 and Fig. 7. In Table $4, V_{\max }$ is the maximum velocity reached in the run, $t_{V_{\max }}$ is the time at which this velocity is reached, $T_{\text {hurricane }}$ the duration for which $v$ is above $33 \mathrm{~m} / \mathrm{s}$ and $T_{\text {storm }}$ the duration for which $v$ is above $17 \mathrm{~m} / \mathrm{s}$. For a very weak vortex $\left(V_{0}=2\right)$, there is hardly any increase in the velocity. For $V_{0}=5 \mathrm{~m} / \mathrm{s}$, there is some amplification with a doubling of the initial strength by 86 hours, followed by decay. For $V_{0}=8 \mathrm{~m} / \mathrm{s}$, more rapid intensification takes place and tropical storm strength is reached within two days, a maximum velocity of $24 \mathrm{~m} / \mathrm{s}$ in 64 hours, followed by slow decay. For $V_{0}$ of $10 \mathrm{~m} / \mathrm{s}$ the behavior is similar in structure to that with $V_{0}=11$ $\mathrm{m} / \mathrm{s}$, except that the maximum velocity just reaches hurricane strength of 33 $\mathrm{m} / \mathrm{s}$. For $V_{0}=12 \mathrm{~m} / \mathrm{s}$, the maximum velocity reached is $41.90 \mathrm{~m} / \mathrm{s}$ and the hurricane is sustained for nearly 5 days. The finite amplitude effect observed in these simulations is similar to the results of Rotunno and Emanuel (1987), Emanuel (1989).

The maximum potential intensity (MPI) theory Emanuel (1988) and Holland (1997) provides a limit on the strength of a hurricane based on the environmental factors such as ambient humidity, SST. We find that that maximum strength reached in the simulations is lower than the value from MPI for similar ambient conditions. For example: for $T_{\text {sea }}$ of 300.5 , a $V_{\max }$ of $45.97 \mathrm{~m} / \mathrm{s}$ is predicted by MPI while our calculation is 41.90 for an initial vortex strength of 12 (Table 4). 


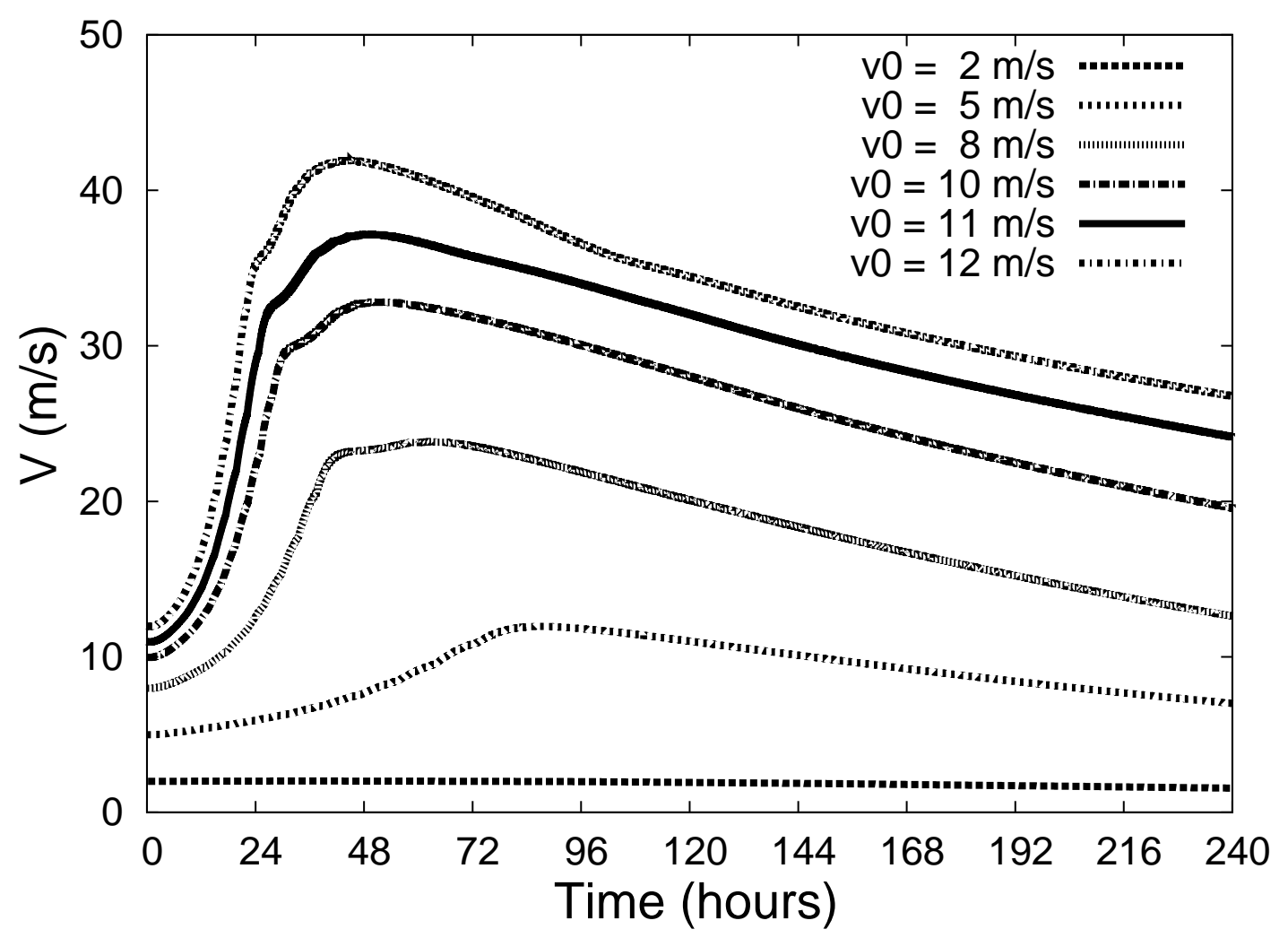

Figure 7: Evolution of full vortices of different initial strengths $\left(v_{0}\right)$ is shown by plotting the maximum azimuthal velocity $(v)$ with time.

The reason for the finite amplitude nature can be understood as follows. In figure 8 , the evolutions of the individual terms (denoted by T1, T2 and T3) on the RHS of equation 1, at a point close to the maxima of azimuthal velocity, for various values of the initial vortex strengths are compared. At this point, T1 $(-u \eta)$ and T3 $\left(D_{v}\right)$ are negative for most part, while T2 $(-w \partial v / \partial z)$ is positive. Vortex amplification is due to T2, which is mainly due to the induced vertical velocity. This tendency is opposed by terms T2, radial advection and T3, the turbulent diffusion term. The relative magnitude of $\mathrm{T} 2$ with respect to $\mathrm{T} 1$ and $\mathrm{T} 3$ determines whether amplification or decay occurs. For small initial velocities $\left(v_{0}=2 \mathrm{~m} / \mathrm{s}\right.$, figure 8 (a)), T1 dominates $\mathrm{T} 2$ and therefore there is only decay of the vortex.

For intermediate velocities $\left(v_{0}=5 \mathrm{~m} / \mathrm{s}\right.$, figure $\left.8(\mathrm{~b})\right)$, after about 24 hours, T2 increases rapidly, since the radial inflow in the boundary layer is of sufficient strength to higher vertical velocities at higher levels. The 

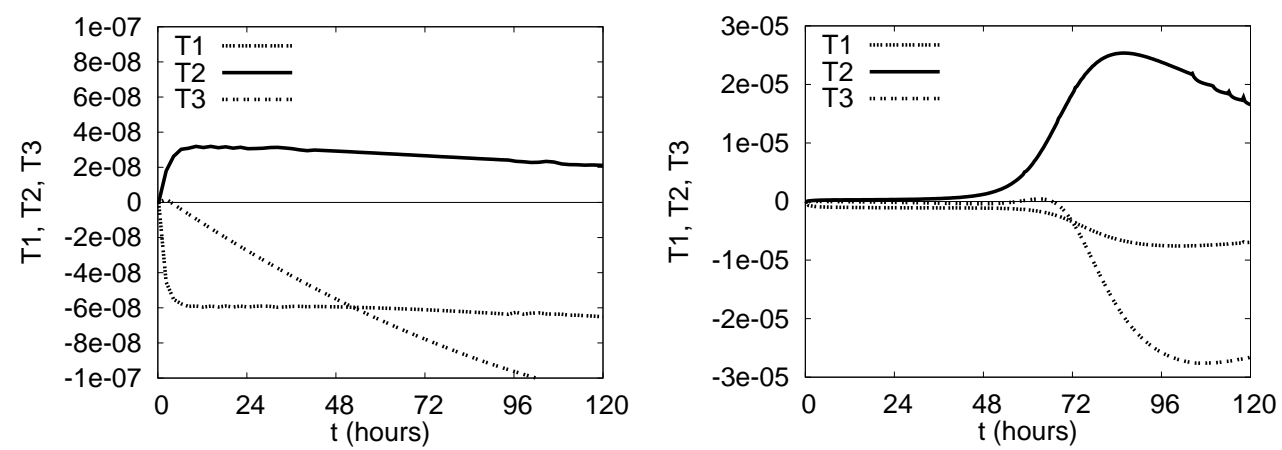

(a) $v_{0}=2 \mathrm{~m} / \mathrm{s}$

(b) $v_{0}=5 \mathrm{~m} / \mathrm{s}$

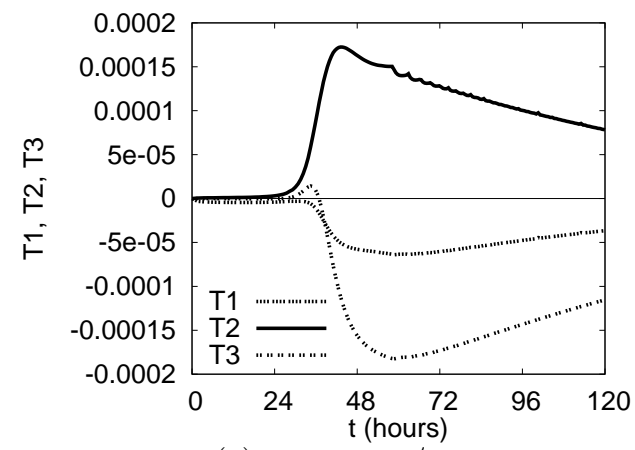

(c) $v_{0}=8 \mathrm{~m} / \mathrm{s}$

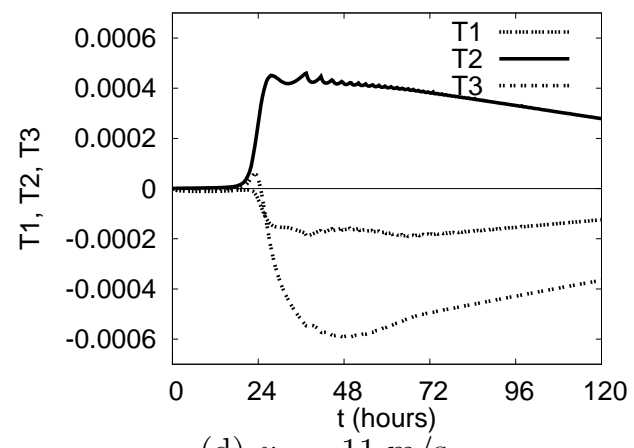

(d) $v_{0}=11 \mathrm{~m} / \mathrm{s}$

Figure 8: Evolution of the terms (T1, T2 and T3) on the RHS of equation 1, at a point close to the maxima of azimuthal velocity, for full vortices of different initial strengths.

corresponding increase in $\mathrm{T} 1$ is not sufficient. It takes some time for $\mathrm{T} 3$ to increase and arrest further growth of the vortex, so only a moderate strength is reached followed by a decay phase.

For velocities above a threshold $\left(v_{0}=8,11 \mathrm{~m} / \mathrm{s}\right.$, figure 8 (c) and (d)), the radial inflow in the boundary layer and the corresponding induced vertical velocities at higher levels close to the centre of the vortex, is sufficiently high and T2 increases exponentially. There is a lag of around 12 hours for T3 to increase. Then a quasi-equilibrium is reached before T3 dominates and causes the vortex to decay. 


\subsection{Parameter sweep experiments}

The effect of the various parameters on the formation is described below. For these comparisons, the case with $V_{0}=11$ was chosen as the control run.

On doubling the domain, keeping the grid size the same, the change in the maximum velocity is marginal (from 32.93 to 33.42 ). This shows that the domain of $500 \mathrm{~km}$ is adequate for the present study.

\begin{tabular}{|l|c|c|c|c|c|c|c|c|}
\hline$r_{\text {vmax }}(\mathrm{m} / \mathrm{s})$ & 50 & 60 & 70 & 80 & 90 & 100 & 125 & 150 \\
\hline$V_{\max }(\mathrm{m} / \mathrm{s})$ & 24.20 & 27.75 & 31.19 & 34.16 & 37.32 & 39.84 & 45.92 & 50.92 \\
\hline
\end{tabular}

Table 5: Effect of the initial radius of maximum winds $\left(r_{v \max }\right)$ on the evolution of the system.

If the radius of maximum wind is decreased from $100 \mathrm{~km}$ to $50 \mathrm{~km}$, (a change of $50 \%$ ), the maximum wind decreases from $39.84 \mathrm{~m} / \mathrm{s}$ to 24.20 (a change of $39.3 \%$ ) (Table 5 ). The reduction is due to the reduced strength of the initial vortex. If the radius of maximum wind is increased to $150 \mathrm{~km}$, (a change of $50 \%$ ), the maximum wind increases to 50.92 (a change of 27.8 $\%$ ). The percentage decrease/increase is lower than that of the change in the $r_{\text {vmax }}$. This suggests that this parameter has a moderate impact.

Amplification depends more on the initial velocity, as seen from table 4 and figure 7 . If both the velocity and the radius of maximum wind are varied, keeping the vorticity constant, the behaviour is similar to that seen in table 4.

\begin{tabular}{|l|l|l|l|l|l|l|l|l|l|}
\hline $\begin{array}{l}T_{\text {sea }} \\
(\mathrm{K})\end{array}$ & 298.5 & 299.0 & 299.5 & 300.0 & 300.5 & 301.0 & 301.5 & 302.0 & 302.5 \\
\hline $\begin{array}{l}V_{\max } \\
(\mathrm{m} / \mathrm{s})\end{array}$ & 30.18 & 30.97 & 33.13 & 35.24 & 37.19 & 40.01 & 43.40 & 46.96 & 48.73 \\
\hline
\end{tabular}

Table 6: Effect of SST on the evolution of the system.

The variation of the maximum velocity reached when the sea surface temperature is varied is shown in table 6 . The non-linear nature of the effect of SST can be seen. A decrease of 2 degrees results in a decrease of $V_{\max }$ of around $7 \mathrm{~m} / \mathrm{s}$, while an increase of 2 degrees results in an increase of $V_{\max }$ of around $11.5 \mathrm{~m} / \mathrm{s}$, When SST is changed, the major impact is on the vertical velocities (term T2), while the radial flow (term T1) is affected marginally (figure 9). The changes in vertical velocity are in turn due to 


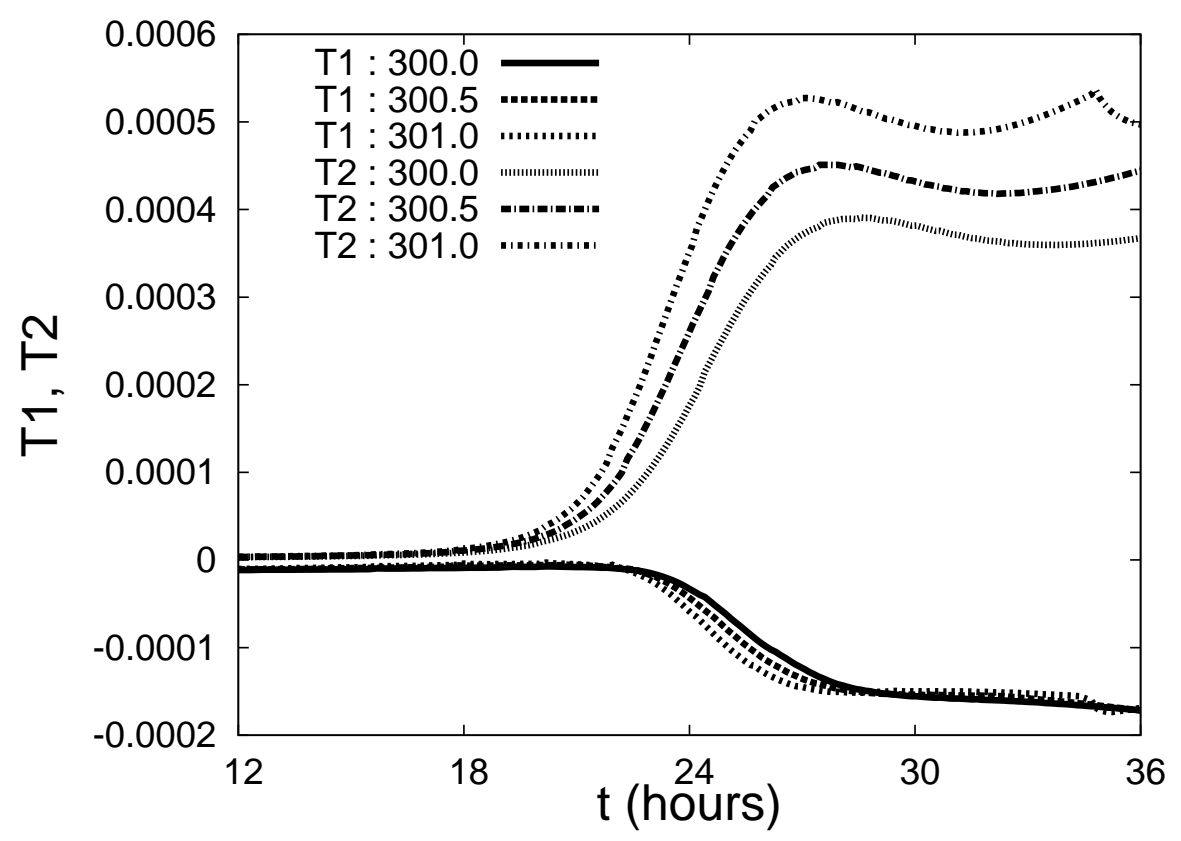

Figure 9: Evolution of the terms T1 and T2 on the RHS of equation 1 for full vortices when the SST is varied.

reduction/increase of fluxes from the sea. This is to be expected as the heating is linked to the sea surface fluxes in our formulation of the model. The large percentage change shows that this is an important parameter.

\begin{tabular}{|l|c|c|c|c|c|c|c|c|}
\hline $\begin{array}{l}\text { Latitude } \\
\left({ }^{o} \mathrm{~N}\right)\end{array}$ & 15 & 16 & 17 & 18 & 19 & 20 & 22.5 & 25 \\
\hline $\begin{array}{l}V_{\max } \\
(\mathrm{m} / \mathrm{s})\end{array}$ & $39.27^{*}$ & $41.85^{*}$ & 42.17 & 39.89 & 38.86 & 37.19 & 34.96 & 33.03 \\
\hline
\end{tabular}

Table 7: Effect of the Coriolis parameter on the evolution of the system. ${ }^{*}$ : Oscillations are observed.

The effect of the Coriolis parameter was studied by varying the base latitude as given in Table 3 . The maximum values of $v$ reached are shown in table 7 . The general trend (from $17 \mathrm{~N}$ ) is that $v_{\max }$ decreases on increasing latitude. This is because an increase in the value of $f$ leads to an increase in the term $T 2$ which tends to oppose the amplification of the vortex. For latitudes 15 and 16 degrees, large fluctuations are observed in the velocity versus time graph. Decreasing the time step, did not change the results 


\begin{tabular}{|l|c|c|c|c|c|c|c|c|}
\hline $\begin{array}{l}\text { Latitude } \\
\left({ }^{o} \mathrm{~N}\right)\end{array}$ & 15 & 16 & 17 & 18 & 19 & 20 & 22.5 & 25 \\
\hline $\begin{array}{l}V_{\max } \\
(\mathrm{m} / \mathrm{s})\end{array}$ & 33.03 & 32.75 & 31.05 & 29.97 & 29.21 & 28.59 & 26.91 & 25.82 \\
\hline
\end{tabular}

Table 8: Effect of the Coriolis parameter on the evolution of the system, with an increased value of the eddy viscosity parameter: $l_{h}=2400$.

indicating that this was not a problem of numerical stability. The reduced inertial stability when the Coriolis parameter is reduced could be the cause. Most other studies like those of Emanuel, Sundqvist and Zhu et al have used the $20 \mathrm{~N}$ as the base latitude, probably for this reason. As a further check, the horizontal eddy viscosity term $\left(K_{H}\right)$ was increased by increasing $l_{h}$ from 2000 to 2400 . This lead to a suppression of the oscillations and a decrease in the maximum velocity reached (from 39.27 to 33.03). With this higher value of $l_{h}$, the $V_{\max }$ decreases monotonically as latitude increases (Table 8).

\subsection{Mid-level vortices}

The mechanism for formation of mid-level vortices is well known and can be explained by the thermal wind relation (Raymond and Jiang, 1990). In a region of the tropics having a large cumulus cloud, stratiform heating takes place in the anvil region due to release of latent heat of condensation. If the region is not saturated, there is re-evaporation of rain in the lower troposphere causing it to cool. Thus a vertical dipole of heating is formed. If this heating pattern is sustained and if the local Rossby radius of deformation is reduced, it results in temperature perturbations having nearly the same spatial pattern. Thermal wind balance then requires the formation of a midlevel vortex. This process is simulated by specifying the heating pattern by the function $\dot{Q}(r, z)=-\sin \left(2 \pi z / z_{\max }\right) \exp \left(-\alpha r^{2}\right)$. The resulting velocity field has a maxima at the middle of the domain $\left(z_{\max } / 2\right)$.

\subsubsection{Decay of mid-level vortices}

To demonstrate the decay of mid-level vortices, simulations were performed keeping all the other parameters same as the previous study but changing the initial condition to that for a mid-level vortex. In the vertical, the velocity maximum was chosen to be at levels 4 and 5 . Above and below this level, the variation of velocity was taken to be $(1 / 2)\left(1.0+\tanh \left(3\left(z_{c}-\right.\right.\right.$ $1 / 2)))$ where $z_{c}$ is the vertical distance measured from this level, normalized 
by the vertical distance to the domain limits (boundary layer height below and $z_{\max }$ above).

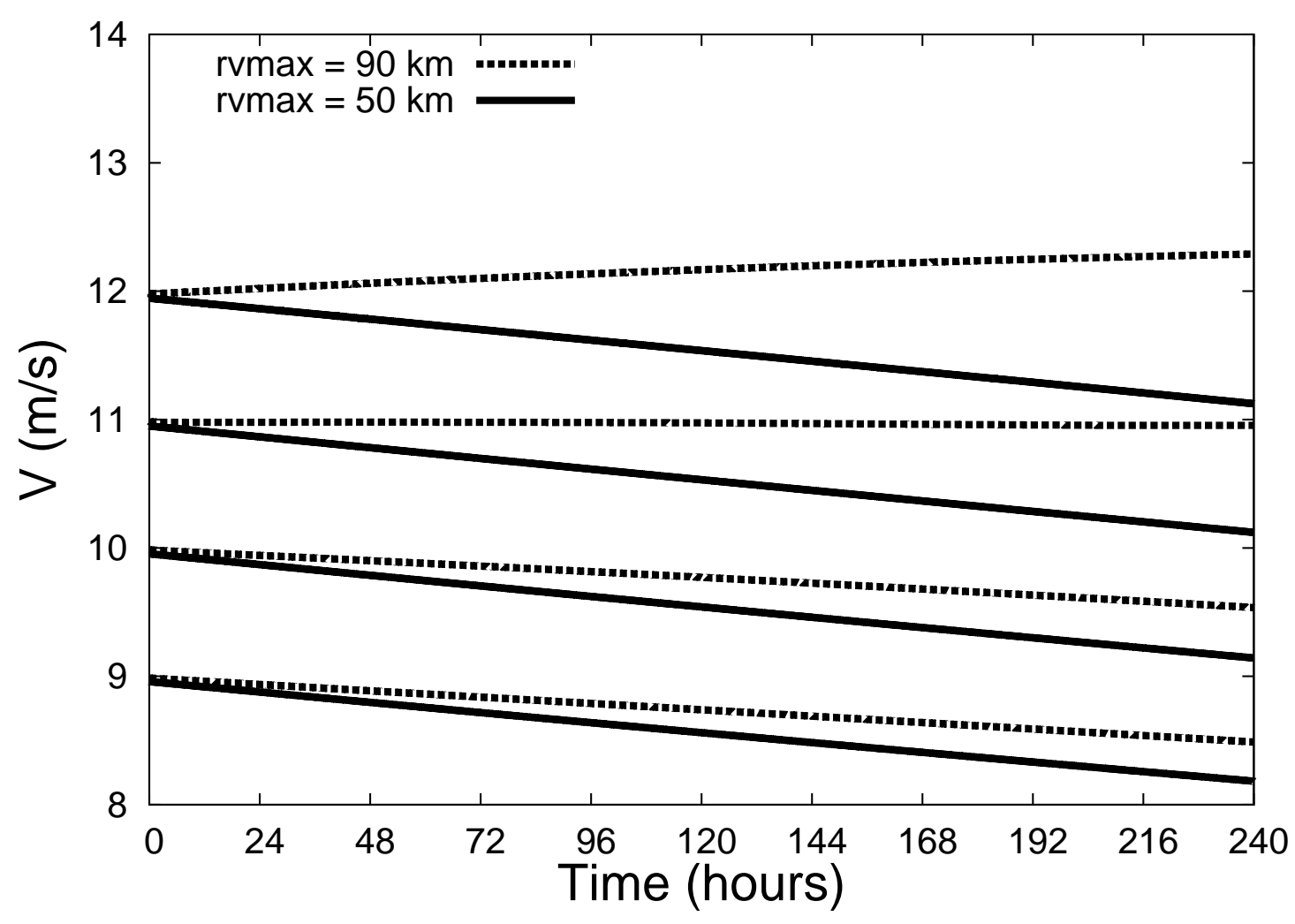

Figure 10: Evolution of mid-level vortices of different initial strengths $\left(v_{0}\right)$ and location of radius of maximum winds $\left(r_{v \max }\right)$ is shown by plotting the maximum azimuthal velocity $(v)$ with time.

The main forcing here is that due to turbulent dissipation which is weak at mid levels since no surface processes are present. In the model, the minimum values of the eddy viscosities were set to $1 \mathrm{~m}^{2} / \mathrm{s}$ in the vertical and $100 \mathrm{~m}^{2} / \mathrm{s}$ in the radial direction. This leads to a very slow spin down. The results are shown in figure 10. For the same vortex strengths, which caused hurricane formation when placed just above the boundary layer, there is either slow decay $\left(V_{0}=10\right)$ or marginal increase $\left(V_{0}=12\right)$. There is slow diffusion of the vorticity causing the surface vortex to grow but the magnitude reached even after 10 days is too small for convection to be triggered. Thus for all practical purposes, the vorticity can be considered constant. 


\subsubsection{Extension to boundary layer}

The present model, being an axisymmetric one, cannot simulate the baroclinic, three dimensional merger of two mid-level vortices. We use the results available in the literature for this problem and study the evolution following a merger by introducing a suitable "merged" vortex. The "merged vortex" is simulated by specifying a mid-level vortex with increased vertical extent. This is due to the existence of an optimum aspect ratio as shown by studies of Reinaud et al (2003).

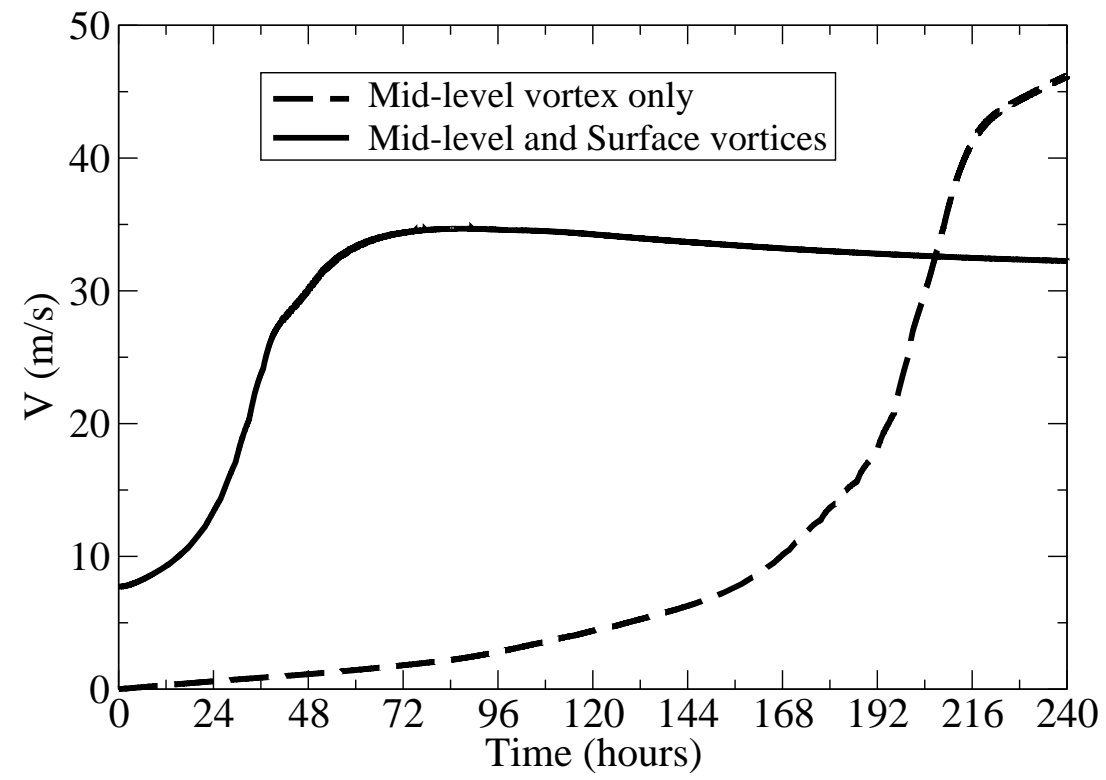

Figure 11: Evolution of the maximum azimuthal surface wind $(\mathrm{m} / \mathrm{s})$ is shown when a) only a mid-level vortex is present (dashed line) and b) a mid-level vortex and a surface vortex vortex are present (solid line). The initial strengths of the mid-level and surface vortices are $V_{\max }=12 \mathrm{~m} / \mathrm{s}$ and $7 \mathrm{~m} / \mathrm{s}$ respectively.

We construct "merged" mesoscale vortices by stretching the vertical profile of velocity. The velocities of the level of maximum velocity and those above are left untouched. The levels below are assigned the values of that of the level above it (of the original profile). The reason for this is that in the simulations of merger of mid-level vortices (Ritchie and Holland, 1997), the increase in the vertical direction is mainly below the initial vortex. The increase above is marginal. 
While considering mid-level vortices, there are two possibilities: i) only a mid-level vortex is present with no surface vorticity and ii) a weak surface vortex is present along with the merged mid-level vortices. Ritchie and Holland (1997) have shown that the presence of a surface vortex typical of a monsoon trough enhances the merger process. This was demonstrated by vortex patch simulations also, where the presence of a large scale depression results in an increase in the distances up to which merger takes place (Venkatesh 2003). Montgomery and Enagonio (1998) simulated a mid-level vortex with peripheral convection and showed that the axisymmetrization process leads to a spin up of a surface vortex. Therefore, simulations were performed for both the cases.

For the second case, an additional surface vortex obtained by extending the tanh velocity profile to the top of the boundary layer was used. The strength of the surface vortex was varied from $2.4 \times 10^{-5}$ to $2.2 \times 10^{-4}$. These values are comparable to the value $\left(23 \times 10^{-5} \sec ^{-1}\right)$ taken by Ritchie and Holland (1997). Results for both the cases are as follows.

\section{Without a surface vortex}

For both the half extended case (stretching of velocity profile one level) and extended case (stretching of velocity profile by two levels), with the surface velocity set to zero, the overall behavior is identical. The reason for this is that vertical diffusion makes the velocity profiles below the vortex to reach a similar state in a very short time for both the cases. The subsequent development is then identical. Initially the mid-level vortex decays slowly and the surface vorticity grows. By 100 hours, the surface vortex is strong enough for surface fluxes and convection to develop. Then there is rapid growth of the surface vorticity and reduction of the mid-level vorticity (due to eddy diffusion). By 150 hours, the surface vortex becomes stronger and intensifies, reaching hurricane intensity by 200 hours (Fig. 11). In reality, it is unlikely that this sequence of events would occur, as the time taken for the surface vortex to amplify (100 hours) is too large. The chief effect of mean winds, which cannot be accounted for in the present model, would be to advect the mid-level vortices over a period of 4-5 days. In simulations by Rogers and Fritsch (2001), the time for vorticity to reach the surface is of the order of a few hours. 
With a surface vortex

The time evolution of velocity for this case is when the surface vorticity is $17 \times 10^{-5}$ is shown in Fig. 11. The surface vortex initiates convection and grows rapidly, overtaking the mid-level vortex within a day. It intensifies to hurricane strengths in 60 hours and a maximum velocity of $35 \mathrm{~m} / \mathrm{s}$ is reached by 75 hours, followed by a gradual decay. The structure of the vortex at steady state is similar to that obtained from the amplification of a full vortex. It should be noted that a full vortex with $V_{0}=8 \mathrm{~m} / \mathrm{s}$ does not intensify into a hurricane. The evolution in this case is closer to that of the full vortex with $V_{0}=12 \mathrm{~m} / \mathrm{s}$. Furthermore there is a longer quasi-steady state.

\begin{tabular}{|c|c|c|c|c|c|c|c|c|c|}
\hline $\begin{array}{c}\text { Surface vorticity } \\
\left(\times 10^{-5} s^{-1}\right)\end{array}$ & 2.4 & 4.8 & 7.3 & 9.7 & 12.2 & 14.6 & 17.0 & 19.5 & 22.0 \\
\hline $\begin{array}{c}t_{\text {hurr }} \\
(\text { hours })\end{array}$ & 178 & 142 & 123 & 112 & 115 & 83 & 60 & 42 & 31 \\
\hline
\end{tabular}

Table 9: Effect of the strength of the surface vortex on the time taken to reach hurricane strength.

The strength of the surface vortex determines the time taken for amplification (see table 9). For weak surface vortices, the time needed to reach hurricane strength is more than five days. For vortices with strength $17 \times 10^{-5} s^{-1}$ or greater, the time scale of amplification is realistic.

\section{Summary and Conclusions}

A simple axisymmetric model has been developed for tropical cyclone related studies. A feature of this model is that it can resolve the vertical structure of vortices which are likely to be present in the early stages of genesis. The model numerics has been validated using the analytical results available for spin-down half lives. For full vortices, studies of the finite amplitude nature, the dependence on various parameters, like sea surface temperature, Coriolis parameter, initial vortex strength have been carried out and these compare well with other simulations of this kind.

The main results from the balanced vortex calculations are the following: If no cloud heating is present, full vortices decay in about 5 days with the decay rate increasing with the initial strength. Mid-level vortices on the other hand decay very slowly and can be assumed to remain nearly steady. With 
cloud heating and sea surface fluxes, full vortices amplify, reach hurricane strength within two days, are in a quasi steady state for around 4-5 days and decay slowly. The finite amplitude nature of this amplification is also evident. While weak vortices decay, those above a threshold $\left(V_{0}=10 \mathrm{~m} / \mathrm{s}\right.$, $r_{v \max }=90 \mathrm{~km}$ ) amplify. The maximum velocity reached and the duration of the hurricane depends on the initial velocity. The sea surface temperature has a crucial role, and the maximum velocity reached depends on small changes to it.

Merged mid-level vortices with an increased vertical extent, but with no vorticity at the surface amplify but not on a realistic time scale. Merged midlevel vortices with an increased vertical extent and with a surface depression of typical strength amplify and reach hurricane strengths.

A novel feature of this paper is that a comparison of evolution of midlevel vortices and full vortices has been done. It is shown that mid-level votices decay if no other effects are present. Also it is shown that if merger is simulated and the strength of the surface vortex increases, there is rapid intensification of these vortices, in a manner similar to full vortices. These results form an important part of the evidence in favour of the authors' model (Venkatesh and Mathew, 2004) for tropical cyclone genesis.

\section{References}

[] Bister, M., Emanuel, K. A., 1997. The genesis of Hurricane Guillermo: TEXMEX analyses and a modeling study. Monthly Weather Review 125, $2662-2682$.

[] Challa, M., Pfeffer, R.L., 1980. Effects of eddy fluxes of angular momentum on the model hurricane development. Journal of the Atmospheric Sciences $37,1603-1618$.

[] Challa, M., Pfeffer, R.L., Zhao, Q., Chang, S.W., 1998. Can eddy fluxes serve as a catalyst for Hurricane and Typhoon formation ?. Journal of the Atmospheric Sciences 55, 2201-2219.

[] Eliassen, A., 1952. Slow thermally or frictionally controlled meridional circulation in a circular vortex. Astrophysica Norvegica 5, 19-60.

[] Eliassen, A., Lystad, M., 1977. The Ekman layer of a circular vortex. A numerical and theoretical study. Geophysica Norvegica 31, 1-16. 
[] Emanuel, K.A., 1986. An air-sea interaction theory for tropical cyclones. Part I: Steady state maintenance. Journal of the Atmospheric Sciences 43, 585-604.

[] Emanuel, K. A., 1988. The maximum intensity of hurricanes, Journal of the Atmospheric Sciences 45, 1143-1155.

[] Emanuel, K.A., 1989. Finite amplitude nature of tropical cyclogenesis. Journal of the Atmospheric Sciences 46, 3431-3456.

[] Emanuel, K. A., 1995. The behavior of a simple hurricane model using a convective scheme based on subcloud layer entropy equilibrium. Journal of the Atmospheric Sciences 52, 3960-3968.

[] Fudeyasu, H., Wang, Y., Satoh, M., Nasuno, T., Miura, H., Yanase, W., 2008. Global cloud-system-resolving model NICAM successfully simulated the lifecycles of two real tropical cyclones. Geophysical Research Letters 35, L22808, doi:10.1029/2008GL036003.

[] Hendricks, E. A., M. T. Montgomery, and C. A. Davis, 2004. The role of "vortical" hot towers in the formation of tropical cyclone Diana (1984). Journal of the Atmospheric Sciences 61, 1209 - 1232.

[] Holland, G. J., 1997. The maximum potential intensity of tropical cyclones. Journal of the Atmospheric Sciences 54, 2519-2541.

[] Houze, R.A., 1993. Cloud Dynamics. Academic Press.

[] Jordan, C., L., 1958. Mean soundings for the West Indies area. Journal of Meteorology 15, 91-97

[] Khalili, A., Basu, A.J., Mathew, J., 1997. A Simpler implicit scheme for computing unsteady incompressible flows. Computational Fluid Dynamics Journal 6, 385-398.

[] Kim, J., Moin, P., 1985. Application of a fractional step method to incompressible Navier-Stokes equations. Journal of Computational Physics 59, 308-323.

[] Montgomery, M.T., Enagonio, J. 1998. Tropical cyclogenesis via convectively forced vortex Rossby waves in a 3-D quasi-geostrophic model. Journal of the Atmospheric Sciences 55, 3176-3207. 
[] Montgomery, M.T., Snell, H.D., Yang, Z., 2001. Axisymmetric Spindown of Dynamics of Hurricane-like vortices. Journal of the Atmospheric Sciences 58, 421-435.

[] Montgomery, M. T., M. E. Nicholls, T. A. Cram, and A. B. Saunders, 2006. A vortical hot tower route to tropical cyclogenesis. Journal of the Atmospheric Sciences 63, 355 - 386.

[] Ooyama, K., 1969. Numerical simulation of the life cycle of tropical cyclones. Journal of the Atmospheric Sciences 26, 3-40.

[] Raymond, D.J., Jiang, Jiang, H., 1990. A theory for long-lived mesoscale convective systems. Journal of the Atmospheric Sciences 47, 3067 - 3077.

[] Reinaud, J.N., Dritschel, D.G., Koudella, C.R., 2003. The shape of vortices in quasi-geostrophic turbulence. Journal of Fluid Mechanics 474, 175-192.

[] Ritchie, E.A., Holland, G.J., 1997. Scale interactions during the formation of typhoon Irving. Monthly Weather Review 125, 1377-1396.

[] Rogers, R.F., Fritsch, J.M., 2001. Surface cyclogenesis from convectively driven amplification of midlevel convective vortices. Monthly Weather Review 129, 605-637.

[] Rotunno, R., Emanuel, K.A., 1987. An air sea interaction theory for tropical cyclones. Part II: Evolutionary study using a nonhydrostatic axisymmetric numerical model. Journal of the Atmospheric Sciences 44, 542-561.

[] Schubert, W.H., Hack, J.J., 1982. Inertial stability and tropical cyclone development. Journal of the Atmospheric Sciences 39, 1687-1697.

[] Schubert, W.H., Hack, J.J., 1983. Transformed Eliassen balanced vortex model. Journal of the Atmospheric Sciences 40, 1571-1583.

[] Sundqvist, 1970. Numerical Simulation of the development of tropical cyclones with a ten level model. Tellus 22, 359-390.

[] Venkatesh, T.N., 2003. A vortex merger theory for tropical cyclone genesis. PhD Thesis, Dept. of Aerospace Engg. Indian Institute of Science, Bangalore, India. 
[] Venkatesh, T.N., Mathew, J., 2004. Prediction of tropical cyclone genesis using a vortex merger index. Geophysical Research Letters 31, L04105.

[] Venkatesh, T.N., 2006. Mesoscale interactions during the genesis and intensification of the 1999 Orissa supercyclone. Mausam 57, 31-36.

[] Wirth, V., 1998. Thermally forced stationary axisymmetric flow on the f-plane in a nearly frictionless atmosphere. Journal of the Atmospheric Sciences 55, 3024-3041.

[] Zhu, H., Smith, R.K., Ulrich, W., 2001. A minimal three dimensional tropical cyclone model. Journal of the Atmospheric Sciences 58, 19241943.

[] Zhu, H., Smith, R.K., 2002. The importance of three physical processes in a minimal three dimensional tropical cyclone model. Journal of the Atmospheric Sciences 59, 1825-1840. 